\title{
Measurement of Complex Fragments and Clues to the Entropy Production from 42-137-MeV/nucleon Ar + Au
}

\author{
B. V. Jacak, G. D. Westfall, C. K. Gelbke, L. H. Harwood, W. G. Lynch, \\ D. K. Scott, H. Stöker, and M. B. Tsang \\ National Superconducting Cyclotron Laboratory, Michigan State University, East Lansing, Michigan 48823 \\ and \\ T. J. M. Symons \\ Lawrence Berkeley Laboratory, University of California, Berkeley, Califormia 94720
}

(Received 22 August 1983)

\begin{abstract}
Intermediate-rapidity fragments with $A=1-14$ emitted from $42-137-\mathrm{MeV} / \mathrm{nu}-$ cleon $\mathrm{Ar}+\mathrm{Au}$ have been measured. Evidence is presented that these fragments arise from a common moving source. Entropy values are extracted from the mass distributions by use of quantum statistical and Hauser-Feshbach theories. The extracted entropy values of $S / A \approx 2-2.4$ are much smaller than the values expected from measured deuteron-to-proton ratios, but are still considerably higher than theoretically predicted values.
\end{abstract}

PACS numbers: $25.70 . \mathrm{Np}$

We report the first systematic measurements of complex nuclear fragments emitted at intermediate rapidities from reactions of $\mathrm{Ar}$ with $\mathrm{Au}$ at 42,92 , and $137 \mathrm{MeV} /$ nucleon, an energy region where only sparse measurements exist. ${ }^{1-3}$ The observed fragments with $A=1-14$ are explained by emission from a common thermal source and the mass distributions are analyzed by use of quantum statistical ${ }^{4}$ and Hauser-Feshbach $^{5}$ theories to deduce the entropy created in the collision. Because it stays constant during the expansion, the extracted entropy may give information about the hot, compressed stage of the system. ${ }^{4,6-10}$ The entropy may provide information on the nuclear equation of state and phase transitions in nuclear matter. ${ }^{7,11-13}$ It has been proposed that the entropy can be deduced from the ratio of deuterons to protons, ${ }^{6}$ but more recent calculations indicate that heavier fragments must be included in the analysis. ${ }^{4}$ Indeed our values are considerably lower than those deduced from a prescription based only on deuterons and protons, but they differ from current theoretical expectations. ${ }^{8}$

The measurements were carried out at the Lawrence Berkeley Laboratory Bevalac using ${ }^{40} \mathrm{Ar}$ beams of 42,92 , and $137 \mathrm{MeV} /$ nucleon with typical beam intensities of $1 \times 10^{7}$ particles/sec, and targets of 80 and $200 \mathrm{mg} / \mathrm{cm}^{2} \mathrm{Au}$. Heavy fragments were measured with two stacks of silicon detectors $(0.1,0.3,5.0 \mathrm{~mm}$ and $0.8,0.8$, $5.0,5.0,5.0 \mathrm{~mm})$. Light particles $\left(p, d, t,{ }^{3} \mathrm{He}\right.$, ${ }^{4} \mathrm{He}$, and ${ }^{6} \mathrm{He}$ ) were measured simultaneously with a telescope consisting of two silicon $\Delta E$ detectors $(0.4$ and $5 \mathrm{~mm})$ and a $10-\mathrm{cm} \mathrm{NaI} E$ detector. All telescopes covered an angular range of $30^{\circ}$ to $130^{\circ}$ in $20^{\circ}$ steps, with isotope resolution for hydrogen through carbon fragments. The energy spectra of the light particles were corrected for reaction losses in the $\mathrm{NaI}$ detector, and the heavy-fragment spectra were corrected for energy loss in the target. The relative normalization between different angles is accurate to $5 \%$, whereas the absolute normalization is known to $20 \%$.

Measured energy spectra of ${ }^{10} \mathrm{Be}$ are shown in Fig. 1. These spectra are typical of fragments with $A>6$; the complete results will be published elsewhere. The error bars reflect statistical errors only. The solid lines are fits to the spectra assuming particle emission from a single moving source. ${ }^{14}$ The source temperature and velocity and the fragment cross section are obtained by fitting the double differential spectra with a relativistic Boltzmann distribution in the rest frame of the source, with use of a leastsquares method. ${ }^{14}$ This parametrization is used to isolate the component of inclusive spectra arising from an intermediate velocity source. Fragments with $A \leqslant 4$ observed at angles $\leqslant 30^{\circ}$ include substantial contributions from the projectile remnant, and are not included in the fits. Low-energy particles which may be evaporated from the target are also excluded. All of the measured data for fragments with $A>4$ are fitted because the thickness of the first heavy-ion detectors was chosen to exclude evaporation products, and measurements at angles $\geqslant 30^{\circ}$ eliminate the heavy projectile fragments. The resulting temperature for each fragment is shown 


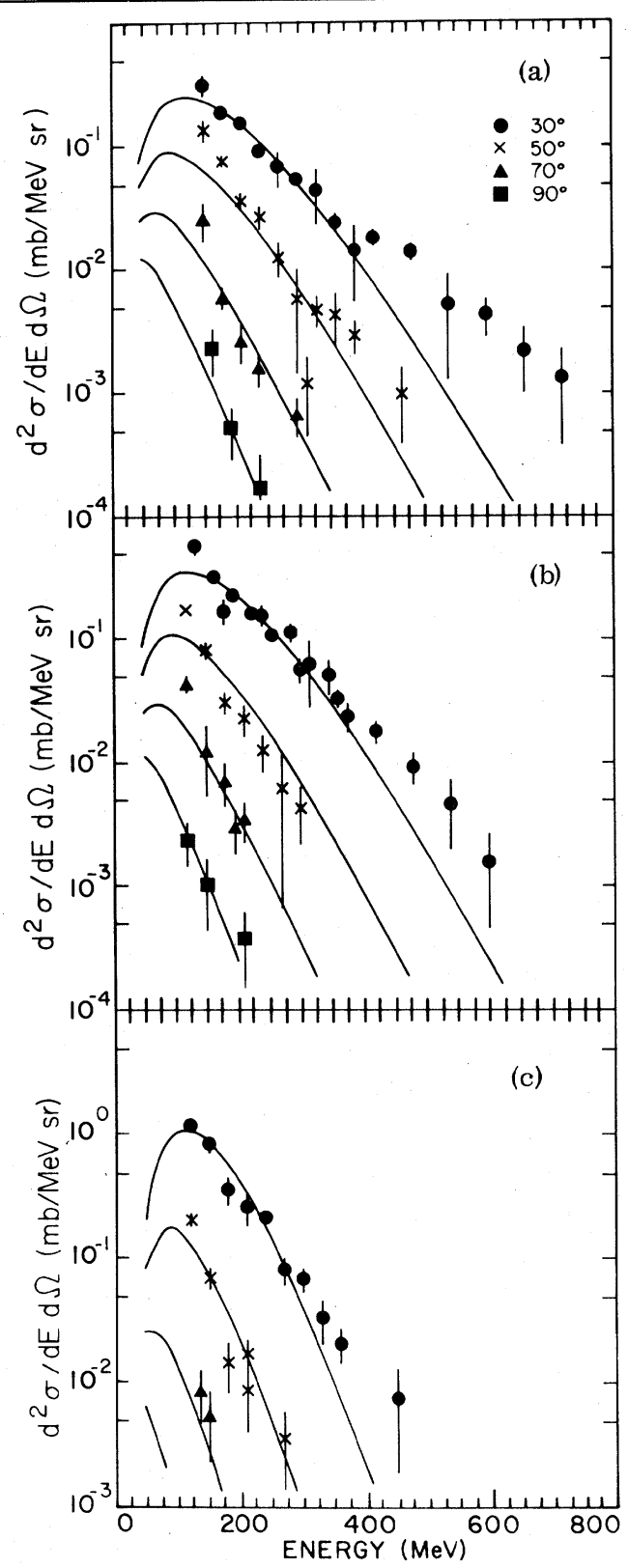

FIG. 1. Energy spectra for ${ }^{10} \mathrm{Be}$ from $\mathrm{Ar}+\mathrm{Au}$ at (a) 137 , (b) 92 , and (c) $42 \mathrm{MeV} /$ nucleon. The solid lines correspond to fits with a single moving source.

in Fig. 2 as a function of the fragment mass.

The similarity of the temperatures over the measured range of fragment masses suggests that the fragments originate from a thermal source and that the same type of source gives rise to the heavy and the light fragments. The source temperature increases with bombarding energy: $T($ ave $)=18,25,35 \mathrm{MeV}$ at $E_{1 \mathrm{ab}}=42,92$, $137 \mathrm{MeV} /$ nucleon, respectively. The fluctuations

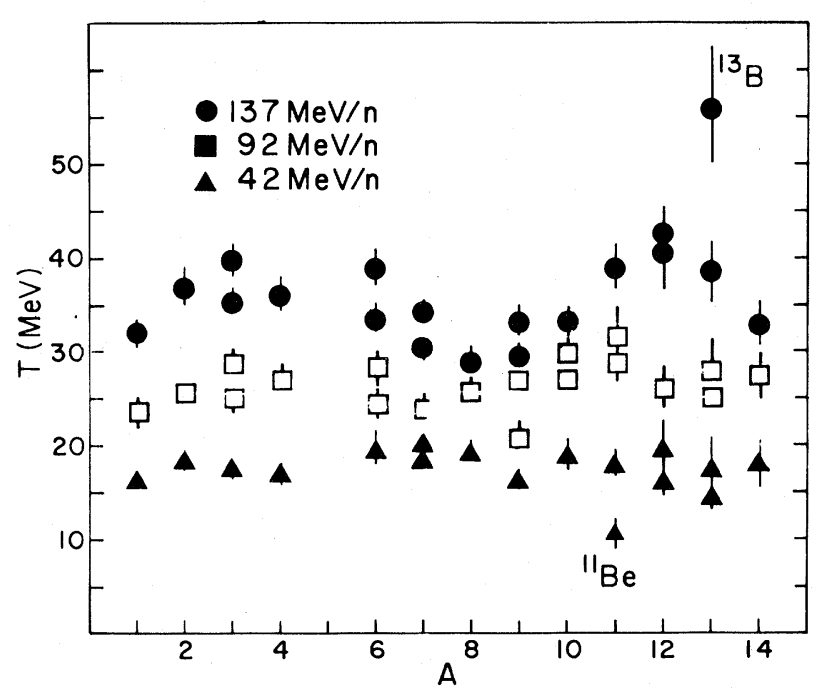

FIG. 2. Temperatures obtained from moving-source fits to the fragment energy spectra, as a function of the fragment mass. Two points for a single mass are shown where two fragments of that mass were observed.

in the temperature extracted from the $137-\mathrm{MeV} /$ nucleon data may be due to the fact that the heavyion telescopes onl y measured particles to 80 $\mathrm{MeV} /$ nucleon, thus sampling only a portion of the intermediate-rapidity data. It would be difficult to account for the production of $A=14$ fragments at intermediate rapidity with models based on . few nucleon-nucleon scatterings; therefore we believe that our results convey evidence for thermalization of the emitting system.

Figure 3(a) shows the relative mass yields as a function of mass. The cross sections are obtained by integrating the moving-source fits in order to focus on the intermediate-rapidity source and exclude particles originating from the projectile and target. The error bars in Fig. 3(a) reflect the error introduced by extrapolation to the unmeasured angles and energies.

The solid histograms show the results of a quantum statistical calculation ${ }^{4}$ of the mass yield. This calculation assumes statistical equilibrium among all accessible quantum states in the late stage of the reaction. The thermodynamic equilibrium state is then fixed by the total isospin, energy, and volume of the system. Ground and excited states with identified spin for nuclei with $A \leqslant 20$ are included in the calculation, as well as known particle-unstable states with $A \leqslant 10$. Pions and delta resonances are also included. In-medium interactions are incorporated via excluded-volume effects. The shape of the cal- 


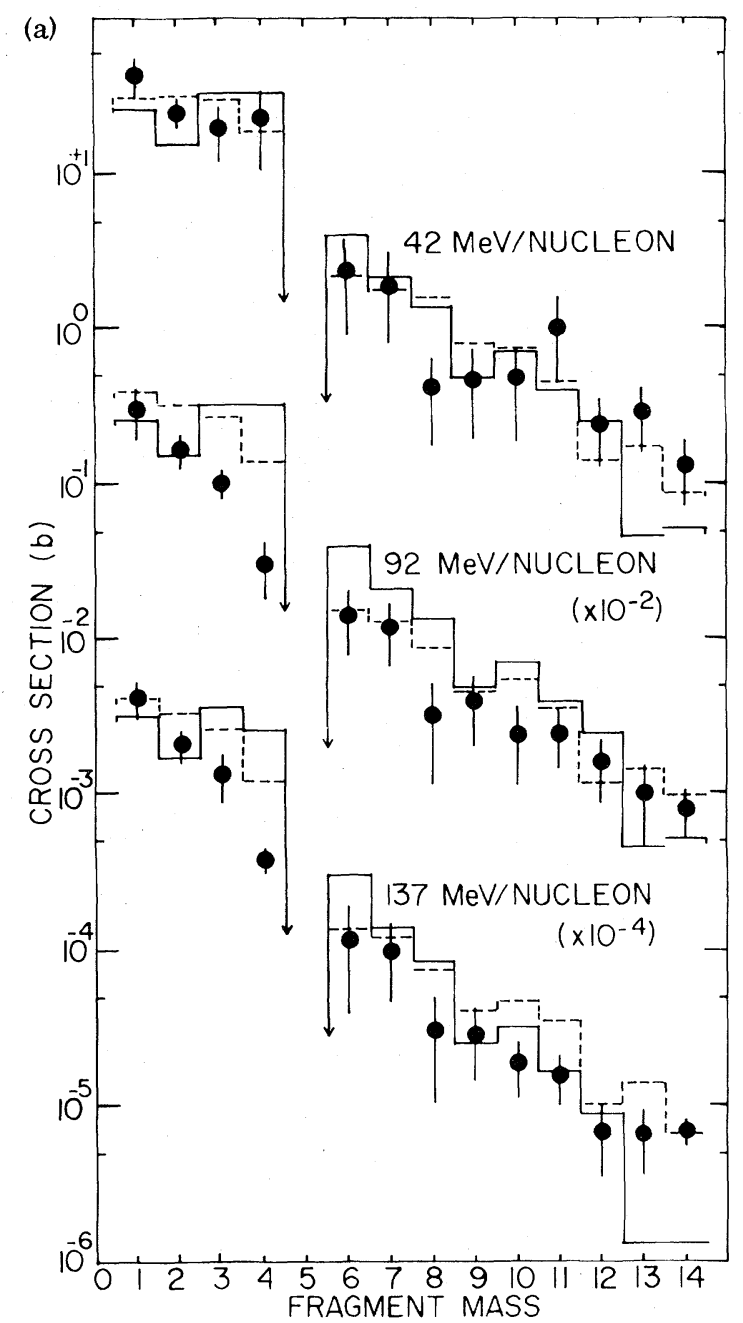

(b)

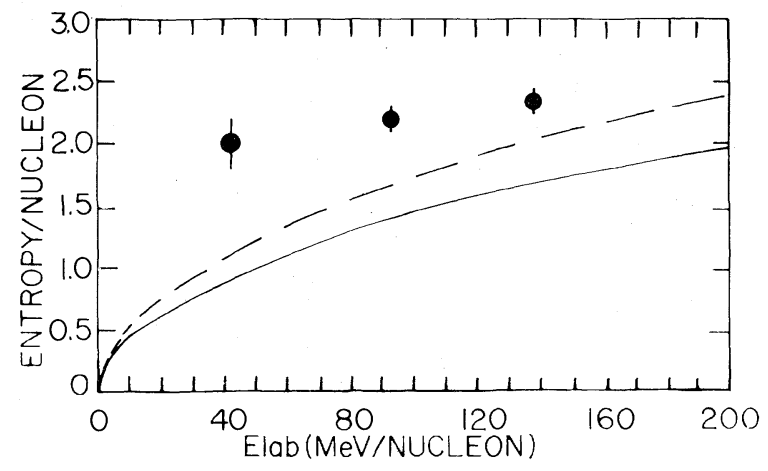

FIG. 3. (a) Production cross sections of fragments with $A=1-14$. The solid and dashed histograms are results of quantum statistical and Hauser-Feshbach calculations, respectively. (b) Entropy extracted from the model fits to the fragment distributions, as a function of bombarding energy. The solid line is the entropy expected from a hydrodynamic calculation, and the dashed line for a viscous fluid (Ref. 8). culated mass yield is well determined by the total entropy in the system for densities of $(0.3-$ $0.7) \rho_{0}$, where $\rho_{0}=0.15 \mathrm{fm}^{-3}$. This allows extraction of the entropy from the experimentally determined mass yield through a least-squares fit of the calculated yields. The histograms are results for $\rho=0.5 \rho_{0}$.

Figure 3(b) shows the entropy values obtained: $S / A=2.0 \pm 0.2,2.2 \pm 0.2$, and $2.35 \pm 0.2$ for $E_{1 \mathrm{ab}}$ $=42,92$, and $142 \mathrm{MeV} /$ nucleon, respectively. The solid line shows the average entropy per nucleon expected for participants with use of a conventional hydrodynamic calculation, and the dashed line the result for a viscous fluid. ${ }^{8}$ Entropies in the range of 4-6 were previously extracted from the observed deuteron-to-proton ratios based on the formula $S / A=3.945-\ln \left(R_{d p}\right),{ }^{6}$ which is applicable for high entropy values only. It has been shown ${ }^{4}$ that $R_{d p}$ rises to a maximum for medium entropies and drops to small values at low entropies as a result of the formation of heavier clusters. The present entropy values are still considerably higher than expected from hydrodynamical calculations. ${ }^{4}$ The theoretical results correspond to an upper limit for the entropy produced because in the calculation the incident matter stops, while at low energies there may be transparency and, hence, no entropy production.

To determine the extent to which the extracted entropy depends on the assumed breakup mechanism, we have performed a calculation based on the Hauser-Feshbach formalism. ${ }^{5}$ These results are shown in Fig. 3(a) as the dashed histograms. In this approach, particles are statistically emitted from an excited nucleus at constant density, and the temperature, charge, and mass evolution of the system are followed. Emission of nuclei in ground and particle-stable excited states as well as unstable states with lifetimes long compared to emission time is included. A spherical initial system with $Z=34$ and $A=82$ is assumed, corresponding to a fireball formed at the most probable impact parameter. The entropy extracted is for the initial system with a level density corresponding to an ideal Fermi gas. Entropies determined by fitting the measured mass yields with respect to $T$ at fixed $\mathscr{E}_{\mathrm{f}}$ were found to be rather independent of $\mathcal{E}_{\mathrm{f}}$ in the range of $24 \leqslant \mathscr{E}_{\mathrm{f}} \leqslant 60 \mathrm{MeV}$. The histograms represent calculations for $\mathscr{E}_{\mathrm{f}}=38 \mathrm{MeV}$, corresponding to an ideal Fermi gas at normal nuclear matter density.

The entropy values deduced from the two very 
different approaches, the Hauser-Feshbach and the quantum statistical models, are consistent to within $S / A$ of 0.2 . This agreement confirms the independence of the entropy determination from assumptions about the breakup dynamics.

In summary, we have measured isotopically separated fragments from Ar on $\mathrm{Au}$ at 42, 92, and $137 \mathrm{MeV} /$ nucleon. The double-differential cross sections indicate that these fragments originate from a single intermediate-rapidity source. The observed mass yields are fitted with two very different models of fragment production which yield consistent values of entropy. These entropies are lower than those based on deuteron-to-proton ratios, but are still considerably larger than theoretical values. This discrepancy may be due to several effects. The detection of a heavy fragment at intermediate rapidity correlates with a high multiplicity, ${ }^{3}$ so that the measured fragments arise from central collisions, but some averaging over impact parameter remains. This averaging has been shown to alter the extracted entropy. ${ }^{15}$ Another possible factor would be a liquid-vapor phase transition, which would cause the expansion to be no longer isentropic and cause additional entropy to be produced. ${ }^{4,7}$

We wish to thank the Bevalac operations staff for their cooperation, and to acknowledge the assistance of Anne Werthmann in the data analysis. This material is based upon work supported by the National Science Foundation through Grant No. PHY 80-17605-01.

${ }^{1}$ K. A. Frankel and J. D. Stevenson, Phys. Rev. C $\underline{23}$, 1511 (1981).

${ }^{2}$ B. Jakobsson et al., Z. Phys. A 307, 293 (1982).

${ }^{3} \mathrm{~J}$. Gosset et al., Phys. Rev. C 16, 629 (1977).

${ }^{4} \mathrm{H}$. Stöcker et al., Nucl. Phys. A400, 63c (1983).

${ }^{5}$ W. A. Friedman and W. G. Lynch, Phys. Rev. C $\underline{28}$, 16 (1983).

${ }^{6} \mathrm{P}$. Siemens and J. Kapusta, Phys. Rev. Lett. $\underline{43}$, 1486 (1979).

${ }^{7}$ H. Stöcker, J. A. Maruhn, and W. Greiner, Phys. Lett. 81B, 303 (1979).

${ }^{8}$ H. Stöcker, Lawrence Berkeley Laboratory Report No. 12302 (unpublished).

${ }^{9}$ G. Bertsch and J. Cugnon, Phys. Rev. C $\underline{24}, 2514$ (1981).

${ }^{10} \mathrm{G}$. Bertsch and P. J. Siemens, Phys. Lett. 126B, 9 (1983)

${ }^{11}$ P. Danielewicz, Nucl. Phys. A314, 465 (1979).

${ }^{12} \mathrm{H}$. Schulz et al., Phys. Lett. $\overline{119 \mathrm{~B}}, 12$ (1982).

${ }^{13}$ M. W. Curtin, H. Toki, and D. K. Scott, Phys. Lett. 123B, 289 (1983).

${ }^{14} \mathrm{G}$. D. Westfall et al., Phys. Lett. 116B, 118 (1982).

${ }^{15}$ H. H. Gutbrod et al., Phys. Lett. 127B, 317 (1983). 